\title{
Seven-Day Salt Loading Impairs Microvascular Endothelium-Dependent Vasodilation without Changes in Blood Pressure, Body Composition and Fluid Status in Healthy Young Humans
}

\author{
Lidija Barića Ines Drenjančevića Anita Matića Marko Stupin $^{a}{ }^{a}$ b \\ Luka Kolar $^{a}$ Zrinka Mihaljevića Helena Lenasi ${ }^{c}$ Vatroslav Šerićd \\ Ana Stupin ${ }^{\mathrm{a}}$ e \\ ${ }^{a}$ Department of Physiology and Immunology, Faculty of Medicine, Josip Juraj Strossmayer \\ University of Osijek, Osijek, Croatia; ${ }^{b}$ Department for Cardiovascular Disease, Osijek \\ University Hospital, Osijek, Croatia; ' Institute of Physiology, Faculty of Medicine, University \\ of Ljubljana, Ljubljana, Slovenia; ${ }^{\mathrm{d} D e p a r t m e n t}$ of Clinical Laboratory Diagnostics, Osijek \\ University Hospital, Osijek, Croatia; ${ }^{e}$ Department of Pathophysiology, Physiology and \\ Immunology, Faculty of Dental Medicine and Health, Josip Juraj Strossmayer University of \\ Osijek, Osijek, Croatia
}

\section{Keywords}

High-salt diet · Microcirculation · Endothelium · Body composition · Renin-angiotensin system

\begin{abstract}
Objectives: We aimed to assess whether a 7-day high-salt (HS) diet affects endotheliumdependent and/or endothelium-independent microvascular function in the absence of changes in arterial blood pressure (BP), and to determine whether such microvascular changes are associated with changes in body composition and fluid status in healthy young humans. Materials and Methods: Fifty-three young healthy individuals (28 women and 25 men) were assigned to a 7-day low-salt diet ( $<3.5 \mathrm{~g}$ salt/day) followed by a 7-day HS diet $(\sim 14 \mathrm{~g}$ salt/day). Skin microvascular blood flow in response to iontophoresis of acetylcholine (ACh) and sodium nitroprusside (SNP) was assessed by laser Doppler flowmetry, and BP, heart rate $(H R)$, plasma renin activity (PRA), serum aldosterone, serum and $24 \mathrm{~h}$-urine sodium, potassium, urea and creatinine levels, together with body composition and fluid status measurement with a 4-terminal portable impedance analyzer were measured before and after diet protocols. Results: BP, HR, body composition and fluid status were unchanged, and PRA and serum aldosterone level were significantly suppressed after HS diet. ACh-induced dilation (AChID) was significantly impaired, while SNP-induced dilation was not affected by HS diet. Impaired AChID and increased salt intake, as well as impaired AChID and suppressed renin-angiotensin system were significantly positively correlated. Changes in
\end{abstract}




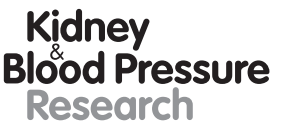

Kidney
Blood Pressure
Research

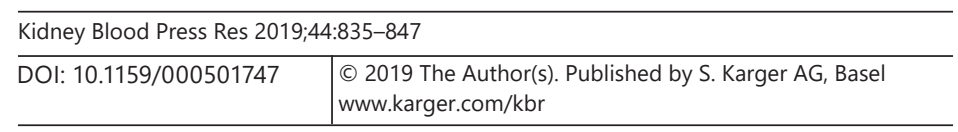

Barić et al.: HS Diet and Microvascular Function

body composition and fluid status parameters were not associated with impaired AChID. Conclusion: 7-day HS diet impairs microvascular reactivity by affecting its endotheliumdependent vasodilation in young healthy individuals. Changes are independent of BP, body composition changes or fluid retention, but are the consequences of the unique effect of HS on endothelial function.

(C) 2019 The Author(s)

Published by S. Karger AG, Basel

\section{Introduction}

Excessive salt intake in most parts of the world presents an important issue because it not only acts as an essential risk factor for the development and progression of hypertension $[1,2]$, but also affects the vascular and endothelial functions even in the absence of changes in blood pressure (BP) [3]. Besides studies on experimental animals, a vast amount of this knowledge was brought by studies on large conductance arteries, such as the study of flowmediated dilation of the brachial artery in healthy population [4-6]. Consistent with other studies [5, 6], we have reported that 7 days of high-salt (HS) intake impaired flow-mediated dilation of the brachial artery, independent of BP changes in healthy individuals [4]. Microcirculation is affected very early in various pathological conditions. Due to its easy accessibility, the skin presents an appropriate site for studying peripheral microcirculation [7]. We have previously reported that a 7-day HS diet impaired forearm skin microvascular reactivity in response to vascular occlusion (in vivo study) [8]. In another study we reported that, after a 7-day HS diet, the mechanisms of vasodilation in response to acetylcholine (ACh) and flow stimulation were switched to non-nitric oxide (NO)-dependent pathways in arterioles isolated from gluteal subcutaneous fat (in vitro study) in healthy women [4], both independent of BP changes.

It is well accepted that sodium is essential for controlling fluid balance in the body, and for maintaining blood volume and BP within a normal range. Hence, it is possible that impaired microcirculatory reactivity may be the consequence of body fluid retention. Contrasting data are published on the effects of HS intake (between 13 and $29 \mathrm{~g}$ of salt per day) on sodium balance and fluid retention $[9,10]$. Either increases in serum sodium concentrations are accompanied by increased fluid retention [11,12] or, in contrast, no further fluid retention was observed with increasing salt intake from an average normal intake ( $8.7 \mathrm{~g} /$ day) to a much higher level (above $17.4 \mathrm{~g} /$ day) in healthy men [9]. Altogether, impaired microvascular function can precede an increase in arterial BP. However, there have been no previous studies on the relation of body composition and fluid status to microvascular reactivity.

Thus, the present study was designed to determine in vivo whether a 7-day HS diet affects endothelium-dependent and/or endothelium-independent vasodilation of forearm skin microcirculation in young healthy adults. In addition, we sought to determine whether such HS intake changes body composition and fluid status of young healthy individuals of both sexes, and if potential changes in body composition/fluid status are associated with microvascular endothelium alterations.

\section{Materials and Methods}

\section{Study Population}

Fifty-three young healthy individuals (28 women and 25 men), aged 18-24 years, were recruited by advertisement at the Faculty of Medicine, University of Osijek to participate in 


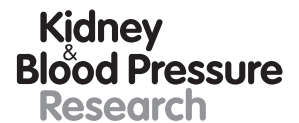

Kidney
Blood Pressure

Research \begin{tabular}{l|l}
\hline Kidney Blood Press Res 2019;44:835-847 \\
\hline DOI: 10.1159/000501747 & $\begin{array}{l}\text { @ 2019 The Author(s). Published by S. Karger AG, Basel } \\
\text { www.karger.com/kbr }\end{array}$ \\
\hline
\end{tabular}

Barić et al.: HS Diet and Microvascular Function

this study. Written informed consent was obtained from each subject. The study protocol and procedures conformed with the standards set by the latest revision of the Declaration of Helsinki and were approved by the Ethical Committee of the Faculty of Medicine, University of Osijek (Cl 602-04/15-08/08; No: 2158-61-07-15-68).

Study was performed in the Laboratory for Clinical and Sport Physiology, Department of Physiology and Immunology at the Faculty of Medicine, University of Osijek. All testing occurred in the morning after an overnight fasting. The focus of this study was on healthy adults; therefore, exclusion criteria included a history of hypertension, coronary artery disease, diabetes, hyperlipidemia, renal impairment, cerebrovascular, and peripheral artery disease. Participants who were obese (body mass index [BMI] $>30 \mathrm{~kg} / \mathrm{m}^{2}$ ) or taking oral contraceptives, antihypertensive agents, anti-inflammatory non-steroidal drugs, steroids or other drugs that could affect the endothelium were also excluded from the study. Women entered the study protocol in the different phases of the menstrual cycle (randomized), which eliminated the effect of sex hormones fluctuation during the menstrual cycle $[4,8]$.

\section{Dietary Salt Perturbation}

This study was a non-randomized controlled experiment in which all subjects were subjected to the same protocol with multiple repeated measurements. During the study all participants had 3 visits to the laboratory - the first visit when entering the study, the second visit after 7 days of low-salt (LS) diet protocol, and the third visit after 7 days of HS diet protocol. All subjects were instructed to first maintain a LS diet, with an intake of $<3.5 \mathrm{~g}$ of salt per day (Dietary Approaches to Stop Hypertension [DASH] eating plan; US Department of Health and Human Services, 2006) for 7 days, which was considered a "wash-out" period. This was followed by 7 days of a HS diet, which implied intake of $3.5 \mathrm{~g}$ of salt per day according to the DASH eating plan and $11.7 \mathrm{~g}$ of salt per day supplemented in the form of a salt powder, which represents a total of about $14.7 \mathrm{~g}$ of salt per day. The amount of salt that represented a HS diet in the present study was set at $14.7 \mathrm{~g}$ of salt per day since such salt intake is not uncommon in everyday life of many individuals (e.g., the average salt intake for adults in Croatia is $11.6 \pm 4.3 \mathrm{~g} /$ day: $13.3 \pm 4.3 \mathrm{~g} /$ day for men and $10.2 \pm 4.2 \mathrm{~g} /$ day for women] [13, $14]$, and when taking into account that similarly designed studies commonly provide 250-400 mmol of $\mathrm{Na}+$ per day (14.6-23.4 g of salt per day), the HS diet used in the present study can be considered as a moderate increase in dietary salt intake. During the whole study protocol, participants had free access to water.

\section{Twenty-Four-Hour Urine Analysis and BP Measurement}

Urine was collected during the last 24-h period of the LS and HS regimes [8]. Twentyfour-hour urine aliquots were analyzed for sodium, potassium, urea, creatinine coefficient, albumin and protein levels at the Department of Clinical Laboratory Diagnostics, University Hospital Osijek. Daily salt intake based on 24-h urinary sodium excretion was calculated using the appropriate formula ( $1 \mathrm{~g}$ salt $[\mathrm{NaCl}]=393.4 \mathrm{mg} \mathrm{Na}=17.1 \mathrm{mmol} \mathrm{Na}$ ).

$\mathrm{BP}$ and heart rate (HR) were measured at the beginning of each visit after a $15 \mathrm{~min}$ rest in a seated position using an automated oscillometric sphygmomanometer (OMRON M3, OMRON Healthcare Inc., Osaka, Japan). The final values of BP and HR were the mean of 3 repeated measurements. Salt resistance was defined as a $\leq 5 \mathrm{~mm}$ Hg change in mean arterial pressure (MAP) determined on LS versus HS diet. Participants were classified as salt-sensitive or salt-resistant after they completed the full protocol. Participants classified as salt-sensitive ( 3 women and 2 men) were excluded from analysis, because they could not be used to test the BP-independent effects of dietary salt (study hypothesis). Forty-eight participants were included in the final data analysis ( 25 women and 23 men). 


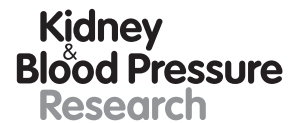

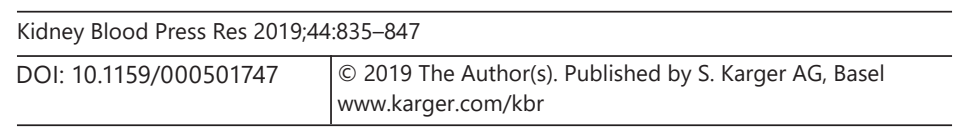

Barić et al.: HS Diet and Microvascular Function

\section{Body Composition and Body Fluid Status Measurement}

BMI and waist-to-hip ratio (WHR) were measured at each study visit. Also, body composition and fluid status were measured using a 4-terminal portable impedance analyzer (Maltron Bioscan 920-II, Maltron International Ltd., Rayleigh, Essex, UK). Empirically derived formulas (the original manufacturer's software) were used to calculate the estimated BMI, fat free mass\%, Fat\% (Fat Mass\%), total body water\% (TBW\%), extracellular water\% (ECW\%), intracellular water\% (ICW\%), plasma fluid (PF), interstitial fluid (IF), and body density $(\mathrm{kg} / \mathrm{L})$.

\section{Assessment of Microvascular Endothelium-Dependent and -Independent Vasodilation}

A noninvasive laser Doppler flowmetry (LDF; MoorVMS-LDF, Axminster, UK) measurement of skin microvascular blood flow in response to the iontophoresis of ACh, which is considered endothelium-dependent vasodilation, and to the iontophoresis of sodium nitroprusside (SNP), which is considered endothelium-independent vasodilation, were performed in each subject according to established guidelines and previously described protocols of our laboratory [15]. The laser probe was attached to the skin of the volar forearm at the same place during each study visit using doubled-sided adhesive discs provided by the manufacturer. Because LDF recordings are very sensitive both to the movement of the examinee and to the movement of the probe or the wire attaching the probe to the LDF device, besides adhesive discs that stabilized the probe to the skin, stationary position of the subject was achieved by a cushion that comfortably stabilized the subject's arm. After baseline recording for $5 \mathrm{~min}$, either the positively charged vasodilator ACh (1\%), or negatively charged SNP (1\%) was applied by previously described protocols [16]. Microcirculatory blood flow in this test was expressed in arbitrary perfusion units and described as area under the curve during baseline flow and during ACh or SNP administration. The final result was expressed as an increase in blood flow following ACh or SNP administration in relation to baseline flow.

\section{Blood Analysis}

A venous blood sample was taken after 30 min resting in a supine position at each visit. Blood samples were analyzed for complete blood count, plasma electrolytes (sodium, potassium, calcium), urea, creatinine, fasting lipid panels (total cholesterol, high-density lipoprotein cholesterol, low-density lipoprotein cholesterol, and triglycerides), fasting blood glucose, and high sensitivity C-reactive protein, using standard laboratory methods. Serum osmolarity was calculated according to the following formula: calculated serum osmolarity $(\mathrm{mOsm} / \mathrm{kg})=2 \mathrm{Na}+$ Glucose + Urea (all in mmol/L). Plasma renin activity (PRA) and serum aldosterone were measured via commercially available ELISA kits (PRA, No. DB52011, IBL International, Germany; Aldosterone Elisa, No. KAPDB450, DIAsource ImmunoAssays, Belgium). All measurements were performed at the Department of Clinical Laboratory Diagnostics, University Hospital Osijek.

\section{Statistical Analysis}

All results are reported as mean \pm SD. The sample size required to show a potentially significant effect was calculated based on preliminary data collected from 10 subjects. To detect differences in primary study endpoints reported in this study (e.g., LDF measurement) with a level of significance of 0.05 and a statistical power of $80 \%$ for paired t-test, the needed sample size is 15 subjects per group. All variables that were measured before and after HS diet protocol were compared using a paired $t$ test. The normality of data distribution was assessed by using the Kolmogorov-Smirnov normality test. The Wilcoxon rank-sum test was used when variables were not normally distributed. The correlations between change of AChID and corresponding parameters (salt intake, PRA, serum aldosterone level) before and 


\section{Research}

Table 1. Baseline participants' characteristics
Barić et al.: HS Diet and Microvascular Function

\begin{tabular}{lc}
\hline Baseline characteristic & Value \\
\hline Demographic data & \\
Number (men/women) & $48(23 / 25)$ \\
Age, years & $21 \pm 2$ \\
BMI, kg/m ${ }^{2}$ & $23.2 \pm 3.4$ \\
WHR & $0.78 \pm 0.05$ \\
BP and HR & \\
Systolic BP, mm Hg & $120 \pm 12$ \\
Diastolic BP, mm Hg & $73 \pm 9$ \\
MPA, mm Hg & $90 \pm 8$ \\
HR, beats per min & $78 \pm 12$ \\
Biochemical parameters & \\
Serum sodium, mmol/L & $138 \pm 2$ \\
Serum potassium, mmol/L & $4.1 \pm 0.3$ \\
Serum calcium, mmol/L & $1.9 \pm 0.2$ \\
Serum urea, mmol/L & $4.7 \pm 1.5$ \\
Serum creatinine, mmol/L & $70 \pm 16$ \\
FBG, mmol/L & $5.0 \pm 0.4$ \\
High sensitivity CRP, mmol/L & $0.96 \pm 1.08$ \\
Cholesterol, mmol/L & $4.48 \pm 0.81$ \\
HDL cholesterol, mmol/L & $1.54 \pm 0.40$ \\
LDL cholesterol, mmol/L & $2.64 \pm 0.61$ \\
Triglycerides, mmol/L & $0.89 \pm 0.37$ \\
Hemoglobin, g/L & $143 \pm 18$ \\
Hematocrit, \% & $42.0 \pm 4.8$ \\
Leukocytes, $\times 109$ /L & $6.7 \pm 2.1$ \\
\hline Values are mean \pm SD. & \\
BMI, body mass index; WHR, waist-to-hip ratio; CRP, C-reactive \\
protein; HDL, high-density lipoprotein; LDL, low-density lipoprotein; \\
BP, blood pressure; HR, heart rate; MPA, mean arterial pressure; FBG, \\
fasting blood glucose. \\
\hline \\
\hline
\end{tabular}

after HS diet were determined by Pearson's or Spearman's correlation tests when appropriate. Multiple linear regression was used to examine the association between changes in AChID and body composition/fluid status parameters following HS diet. $p<0.05$ was considered statistically significant. SigmaPlot, version 11.2 (Systat Software, Inc., Chicago, IL, USA) was used for statistical analysis.

\section{Results}

Participants' clinical characteristics are presented in Table 1. All participants were lean, normotensive, with normal renal function, complete blood count, fasting blood glucose and fasting lipid panels within normal limits. All participants completed a 2-week dietary salt perturbation; the first week of LS diet which was a "wash-out" period and the second week of HS diet.

Effects of Dietary Salt Perturbation on Hemodynamic and Biochemical Parameters

Serum sodium increased, but calculated serum osmolarity did not change following a 7-day HS diet (Table 2). There was no significant difference in other serum electrolytes (potassium, calcium), hemoglobin, hematocrit or in urea and creatinine levels measured before and after HS diet protocol (Table 2). As expected, 24-h urinary sodium excretion as 
Table 2. Hemodynamic and biochemical responses to dietary salt perturbation

\begin{tabular}{lcc}
\hline & LS & HS \\
\hline Biochemical parameters & & $138 \pm 3^{*}$ \\
Serum sodium, mmol/L & $137 \pm 2$ & $4.1 \pm 0.3$ \\
Serum potassium, mmol/L & $4.1 \pm 0.3$ & $2.5 \pm 0.3$ \\
Serum calcium, mmol/L & $2.4 \pm 0.1$ & $4.5 \pm 1.2$ \\
Serum urea, mmol/L & $4.4 \pm 1.3$ & $72 \pm 15$ \\
Serum creatinine, mmol/L & $74 \pm 16$ & $140 \pm 20$ \\
Hemoglobin, g/L & $141 \pm 19$ & $0.413 \pm 0.052$ \\
Hematocrit & $0.418 \pm 0.052$ & $285.6 \pm 5.6$ \\
Calculated serum osmolality, mOsm/kg & $283.8 \pm 5.3$ & $2.20 \pm 1.29^{*}$ \\
PRA, ng/mL/h & $4.65 \pm 3.27$ & $72 \pm 63^{*}$ \\
Serum aldosterone, pg/mL & $170 \pm 116$ & $1,468 \pm 563$ \\
24 h urine volume, mL & $1,321 \pm 549$ & $250 \pm 95^{*}$ \\
24 h urine sodium, mmol/dU & $107 \pm 45$ & $53 \pm 26$ \\
24 h urine potassium, mmol/dU & $45 \pm 21$ & $327 \pm 185$ \\
24 h urine urea, mmol/dU & $296 \pm 138$ & $179 \pm 63$ \\
4 h urine creatinine coefficient, $\mu$ mol/24 h/kg & $9.0 \pm 10.0$ \\
24 h urine albumin, mg/dU & $177 \pm 49$ & $113 \pm 60$ \\
24 h urine proteins, mg/dU & $9.1 \pm 8.3$ & $14.6 \pm 5.5^{*}$ \\
Calculated daily salt intake, g/day & $104 \pm 64$ & $117 \pm 12$ \\
Bnd HR & $6.3 \pm 2.6$ & $72 \pm 7$ \\
Diastolic BP, mm Hg & & $87 \pm 8$ \\
MAP, mm Hg & $116 \pm 12$ & $72 \pm 9$ \\
HR, beats per min & $73 \pm 7$ & $87 \pm 8$ \\
\hline
\end{tabular}

Values are mean $\pm \mathrm{SD}$.

$* p<0.05$ before HS vs. after HS.

LS, low salt; HS, high salt; PRA, plasma renin activity; BP, blood pressure; HR, heart rate; MAP, mean arterial pressure.

Table 3. Body composition and fluid status changes to dietary salt perturbation

\begin{tabular}{lcc}
\hline & LS & HS \\
\hline BMI, $\mathrm{kg} / \mathrm{m}^{2}$ & $23.26 \pm 3.27$ & $23.58 \pm 3.38$ \\
WHR & $0.77 \pm 0.06$ & $0.77 \pm 0.06$ \\
$\mathrm{FFM}, \%$ & $82.1 \pm 7.2$ & $80.3 \pm 8.0$ \\
Fat, \% & $17.9 \pm 7.2$ & $19.7 \pm 8.0$ \\
TBW, $\%$ & $62.4 \pm 7.9$ & $58.8 \pm 11.8$ \\
ECW, \% & $43.5 \pm 4.2$ & $43.2 \pm 2.9$ \\
ICW, \% & $56.5 \pm 4.2$ & $56.8 \pm 2.9$ \\
PF, L & $3.81 \pm 0.90$ & $3.76 \pm 0.94$ \\
IF, L & $13.32 \pm 3.14$ & $13.16 \pm 3.30$ \\
Body density, $\mathrm{kg} / \mathrm{L}$ & $1.058 \pm 0.016$ & $1.054 \pm 0.019$ \\
\hline
\end{tabular}

Values are mean \pm SD.

LS, low salt; HS, high salt; BMI, body mass index; WHR, waist-to-hip ratio; FFM, fatfree mass; TBW, TBW, total body water; ECW, extracellular water; ICW, intracellular water; PF, plasma fluid; IF, interstitial fluid. 
Fig. 1. 24-h urinary sodium excretion and MAP. Despite a significant increase in 24-h urinary sodium excretion during a 7-day HS diet, MAP did not change, confirming that participants were salt-resistant. * $p<0.05$ LS versus HS. LS, low-salt; HS, high-salt; MAP, mean arterial pressure.
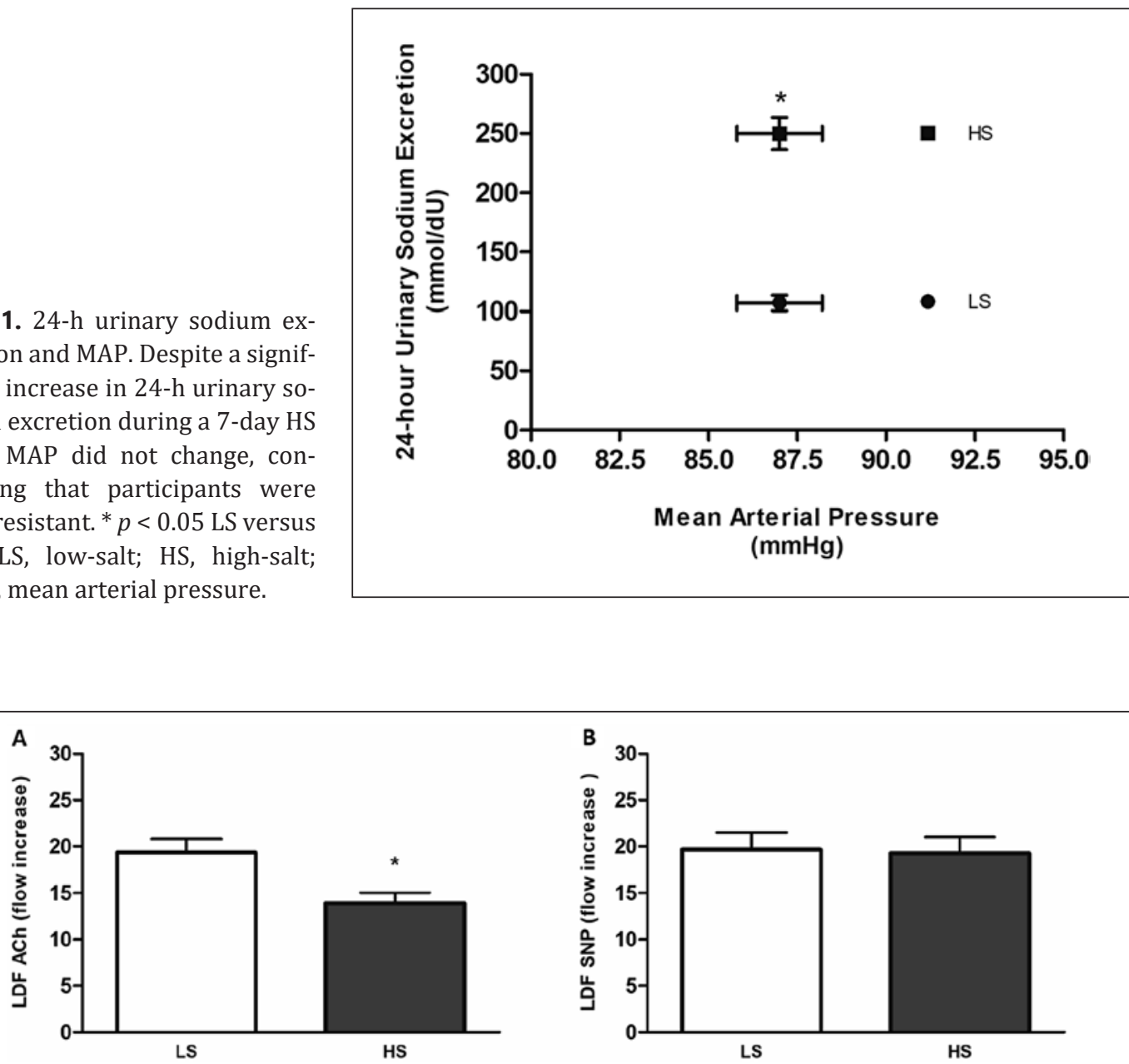

Fig. 2. Endothelium-dependent (A) and endothelium-independent vasodilation (B) of forearm skin microcirculation during HS diet. AChID (endothelium-dependent) was significantly impaired after a 7-day HS diet compared to corresponding values obtained after a LS diet. SNPID (endothelium-independent) did not differ between LS and HS diets. * $p<0.05$ LS versus HS. LDF, laser Doppler flowmetry; ACh, acetylcholine; SNP, sodium nitroprusside; LS, low salt; HS, high salt.

well as calculated daily salt intake significantly increased (Table 2), while PRA and serum aldosterone level were significantly suppressed during the HS diet (Table 2). HS diet did not significantly change the 24-h urinary total volume, creatinine coefficient, potassium, urea, albumin, and protein excretion (Table 2). Values of systolic BP, diastolic BP and MAP during LS diet did not differ compared to the HS diet values (Table 2). Based on MAP, all subjects included in data analysis were characterized as salt-resistant (see Method section), as indicated by a large increase in 24-h urinary sodium excretion (and calculated daily salt intake) during the HS diet without a concomitant change in MAP (Fig. 1).

\section{Effects of Dietary Salt Perturbation on Body Composition and Body Fluid Status}

Body composition and fluid status responses to dietary salt perturbation are presented in Table 3. Seven-day HS diet did not induce any significant change in BMI, WHR, fat free mass \%, Fat\%, TBW\%, ECW\%, ICW\%, PF, IF or body density in young healthy population (Table 3). 

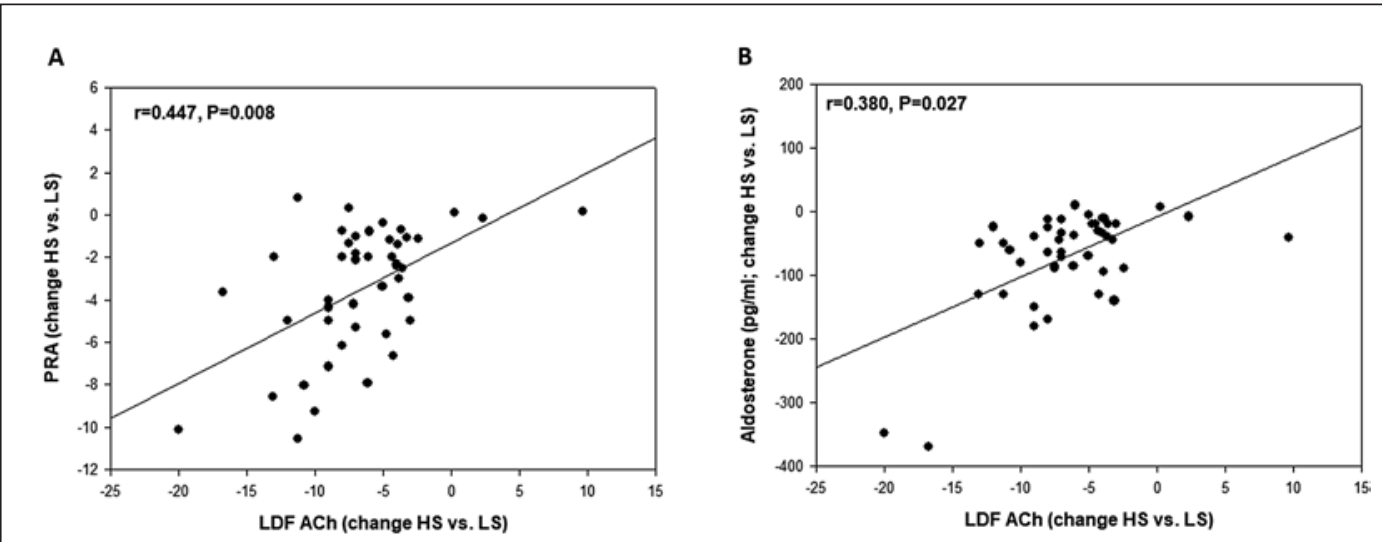

Fig. 3. Correlation between impaired AChID and suppression of RAS during HS diet. There was significant moderate correlation between impaired AChID and PRA suppression (A), and weak but significant correlation between impaired AChID and serum aldosterone level suppression (B) following a HS diet in the study population. LDF, laser Doppler flowmetry; ACh, acetylcholine; LS, low salt; HS, high salt; PRA, plasma renin activity.

Effects of Dietary Salt Perturbation on Body Skin Microvascular Endothelium-Dependent and Endothelium-Independent Vasodilation

Although LDF does not provide an absolute measurement of blood flow and cannot be used to compare absolute values of blood flow between individuals or within an individual at different times, baseline forearm skin microvascular flow values did not differ before and after HS diet protocol (baseline $1 \mathrm{~min}$ area under the curve before HS $548 \pm 245$ vs. after HS $589 \pm 215 ; p>0.05$ ), which indicates consistency and reproducibility of our experimental design. Seven-day HS diet decreased ACh-induced dilation (AChID) of forearm skin microcirculation by approximately 32\% (LDF ACh flow increase LS $21 \pm 15$ vs. HS $14 \pm 6, p<0.05$; Fig. 2A). In contrast to AChID, SNP-induced dilation (SNPID) did not differ between LS and HS diet measurements (LDF SNP flow increase LS $20 \pm 8$ vs. HS $19 \pm 7, p>0.05$; Fig. 2B).

\section{Correlation between Impaired AChID and Salt Intake/Renin-Angiotensin System}

Suppression/Body Composition and Fluid Status Changes following a HS Diet

A significant moderate correlation was observed between AChID and increased salt intake in young healthy participants $(r=-0.507, p=0.003)$. There was also a significant moderate correlation between impaired AChID and PRA suppression $(r=0.447, p=0.008$; Fig. 3A), and a weak but significant correlation between impaired AChID and serum aldosterone level suppression ( $r=0.380, p=0.027)$ following HS diet in the study population (Fig. 3B). A multiple linear regression model to predict changes in AChID from measured body composition and fluid status parameters (body mass, WHR, Fat\%, TBW\%; with other variables eliminated due to multicollinearity) showed that measured changes in body composition and fluid status parameters were not significantly associated with impaired AChID following HS diet in the study population $\left(r^{2}=0.493, p>0.05\right)$.

\section{Discussion}

The salient finding of the present study is that 7 days of a HS diet significantly impaired endothelium-dependent vasodilation (AChID), but not endothelium-independent vasodilation (SNPID) of forearm skin microcirculation in young healthy participants (Fig. 2), without 
affecting MAP (Fig. 1). Furthermore, suppressed PRA and serum aldosterone level significantly correlated with impaired AChID of forearm skin microcirculation (Fig. 3a, b), indicating that suppression of the renin-angiotensin system (RAS; and consequently ANG II levels) may be causal in the development of microvascular dysfunction, independent of the arterial BP levels. We did not observe any significant change in body composition and fluid status following the 7-day HS diet in healthy participants (Table 3).

Endothelium dysfunction is considered to be a precursor and the earliest detectable outcome of cardiovascular diseases [17], the leading cause of mortality and a major cause of morbidity in the world, with 3.9 million deaths in Europe and over 1.8 million deaths in the European Union [18]. As a result of large-scale intervention trials (INTERSALT, TOPH, DASH Sodium Trial) that provided robust evidence about the effect of salt restriction on reduction of BP and cardiovascular event rate [19-21], dietary salt restriction continues to be an important component in lowering BP and treating hypertension. However, in the last 15 years, a body of evidence in animal model studies demonstrated that increased dietary salt intake has an impact on vascular and endothelial function, independent of BP changes [2224]. Some of the mechanisms have been elucidated, such as a profound reduction in nitric oxide bioavailability occurring with HS dietary intake [22-24], or shifts in the metabolisms of arachidonic acid [24]. However, even though a number of previous clinical trials aimed to examine the effect of dietary salt perturbation on endothelial function (besides its effect on $\mathrm{BP})$, these studies have not been able to separate the effects of HS diet and BP on endothelial function, since they were predominantly performed on hypertensive patients. Thus, it was necessary to start investigating the vascular responses to sodium loading (and/or restriction) in healthy, normotensive individuals as well.

Besides the importance of conductance vessels damage in the etiopathogenesis of cardiovascular diseases $[4-6,25,26]$, the impairment of microvascular function can also lead to deleterious end-organ damage and cardiovascular system failure (such as in untreated malignant hypertension, stroke, and sepsis) [7]. Our previous results indicated that vasoconstrictor metabolites of cyclooxygenase (COX) enzymes, especially COX-1, have a significant role in the development of impaired microvascular reactivity in response to vascular occlusion in healthy individuals on a HS diet [8]. Furthermore, we have also reported that vasodilation of arterioles isolated from gluteal subcutaneous fat in response to ACh and flow-induced dilation (in vitro) following a 7-day HS diet were no longer NO-dependent, but that COX and cytochrome P450 vasodilator mediators took part in this vasodilation [4]. However, in the present study we demonstrated, for the first time, that peripheral microvascular endothelialdependent vasodilation, but not endothelial-independent vasodilation, declines in response to a 7-day HS diet in young healthy salt-resistant individuals of both sexes. To date, there is only one more study demonstrating that a 7-day HS intake reduced peripheral microvascular function (measured as reduced red blood cell flux during local heating-induced vasodilation using LDF) independent of BP in normotensive adults [27], which is in concordance with our results.

A growing body of evidence indicates that chronically suppressed RAS and low ANG II levels in rats fed a HS diet have deleterious effects that are almost identical to those occurring at pathologically elevated ANG II levels $[23,28,29]$. These include impaired vascular relaxation in different vascular beds in response to a number of endothelium-dependent and -independent vasodilator stimuli, reduced NO bioavailability, elevated levels of oxidative stress, and decreased vascular antioxidative capacity [23, 30]. The importance of physiological ANG II levels for maintaining normal vascular function was supported by a number of studies showing that vascular relaxation in response to various vasodilator stimuli following a HS diet is restored by continuous IV infusions of suppressor dose of ANG II [31, 32]. However, the potential link between RAS inhibition and disturbed vascular/endothelial function in 


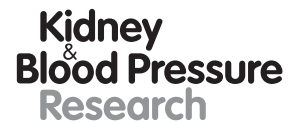

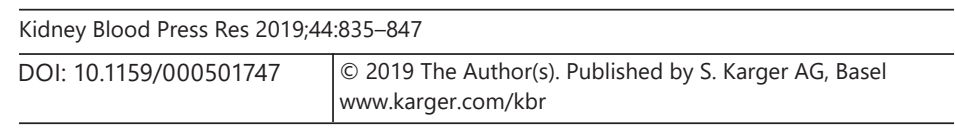

Barić et al.: HS Diet and Microvascular Function

healthy humans is not sufficiently investigated. Accordingly, we have earlier demonstrated that 7 days of ANG II receptors 1 (AT1) blockade in young healthy women on a LS diet significantly increased plasma levels of the stable TXA2 metabolite, suggesting that crosstalk between RAS and production of arachidonic acid vasoconstrictor metabolites may be important in regulating vascular function during HS loading [33]. In the present study, we have found a significant correlation between impaired AChID and suppressed PRA and serum aldosterone levels following a HS diet in young healthy persons (Fig. 3a, b). Thus, it is possible that RAS suppression as a result of a HS diet has an important role in reducing AChID directly and/or via increased oxidative stress level due to disrupted antioxidant defense (or some other mechanism). The latter possibility remains to be investigated in future human studies.

The correlation between daily salt consumption and changes in body composition and/ or fluid status is still an area with limited evidence. In healthy normotensive individuals, an increase in total body weight was observed following a HS diet, without effects on cardiac function (in particular, diastolic function) or cardiac output, suggesting that the increase in total body weight may predominantly reflect an expansion of extracellular volume rather than an increase in intra-vascular volume [34]. To our knowledge, the present study using a 4-terminal portable impedance analyzer is the first controlled interventional experimental study that investigated the effect of a 7-day HS diet (following a LS "wash-out" period) on body composition and fluid status in healthy individuals. We did not observe any significant change either in total body weight or fat mass, or in total body water or particular water compartments (ICW, ECW, PF or IF) following a HS diet in healthy lean participants. This is in concordance with other studies $[9,10]$, for example, increase in daily salt intake from the average normal (2.8 $\mathrm{mmol} \mathrm{NaCl} / \mathrm{kg}$ body mass per day) to a much higher level $(7.7 \mathrm{mmol}$ $\mathrm{NaCl} / \mathrm{kg}$ body mass per day) showed that sodium was retained but without fluid retention, suggesting that the majority of sodium was stored in an osmotically inactive form [9]. There is some experimental evidence that tissues, in particular skin and muscle, can store sodium. However, those observations were made in severe diseases with significantly impaired sodium handling capacity (e.g., primary and secondary hyperaldosteronism, end-stage renal disease, refractory hypertension etc.) [35]. Additionally, there is currently no experimental evidence that skin sodium accumulation has any relationship to endothelial function [35-38]. Excessive sodium plasma concentrations may cause alterations in the endothelial glycocalyx (negatively charged surface rich in water and anionic glycosaminoglycans) [39, 40], as investigated by the Oberleithner group. Furthermore, it has been demonstrated that damaged endothelial glycocalyx could be related to altered endothelial function due to reduction in NO release $[39,40]$. However, the utilized model of salt accumulation in endothelial cells is based on aldosterone effects on sodium retention and is opposite to the effects of dietary salt intake on aldosterone, where aldosterone is suppressed with a HS diet [41]. Taken together, the underlying regulation of such alternative sodium and fluid retention following HS dietary intake remains to be elucidated, particularly since an inability to store osmotically inactive sodium may underlie the pathophysiology of particular diseases related to the salt overconsumption in the Western world.

In conclusion, the results of this study indicate that microvascular changes induced by a 7-day HS diet are independent of body composition changes or fluid retention (just as they are BP-independent), but are a consequence of the unique effect of HS intake (and consequently suppressed RAS) on endothelial function.

\section{Perspectives}

One week of HS diet reduced endothelium-dependent vasodilation (AChID), but not endothelium-independent vasodilation (SNPID) of forearm skin microcirculation in young healthy participants. Observed microvascular reactivity impairment was independent of BP, 
body composition or fluid status changes, but associated with increased salt intake and suppressed RAS. The present findings provide the foundation for further investigations into the exact mechanisms (e.g., RAS inhibition, increased oxidative stress level, changes in sympathetic activity, and/or endothelial-leukocyte activation) mediating microvascular endothelial alterations during HS loading in healthy individuals.

\section{Statement of Ethics}

Subjects have given their written informed consent.

The study protocol has been approved by the Ethical Committee of the Faculty of Medicine, University of Osijek (Cl: 602-04/15-08/08; No.: 2158-61-07-15-68).

\section{Disclosure Statement}

The authors have no conflicts of interest to declare.

\section{Funding Sources}

The present study was supported by Croatian Science Foundation under the project \#IP-2016-06-8744 Interaction of Renin-Angiotensin and Adrenergic System in Oxidative Stress Induced Endothelial Activation (RAS-AdrenOX).

\section{Author Contributions}

L.B., I.D., and A.S.: were involved in formulating the research question(s), designing and conducting the study, gathering, analysis and interpretation of data, drafting the article and revising it critically. A.M., M.S., L.K., and Z.M.: were involved in carrying out the study, assembly, analysis and interpretation of data, drafting the article and revising it critically. H.L. and V.S.: were involved in designing the study, analysis and interpretation of data, drafting the article and revising it critically.

\section{References}

1 Bragulat E, de la Sierra A. Salt intake, endothelial dysfunction, and salt-sensitive hypertension. J Clin Hypertens (Greenwich). 2002 Jan-Feb;4(1):41-6.

2 Drenjančević-Perić I, Jelaković B, Lombard JH, Kunert MP, Kibel A, Gros M. High-salt diet and hypertension: focus on the renin-angiotensin system. Kidney Blood Press Res. 2011;34(1):1-11.

3 Weinberger MH. Salt sensitivity is associated with an increased mortality in both normal and hypertensive humans. J Clin Hypertens (Greenwich). 2002 Jul-Aug;4(4):274-6.

4 Cavka A, Jukic I, Ali M, Goslawski M, Bian JT, Wang E, et al. Short-term high salt intake reduces brachial artery and microvascular function in the absence of changes in blood pressure. J Hypertens. 2016 Apr;34(4):676-84.

5 Lennon-Edwards S, Ramick MG, Matthews EL, Brian MS, Farquhar WB, Edwards DG. Salt loading has a more deleterious effect on flow-mediated dilation in salt-resistant men than women. Nutr Metab Cardiovasc Dis. 2014 Sep;24(9):990-5.

6 DuPont JJ, Greaney JL, Wenner MM, Lennon-Edwards SL, Sanders PW, Farquhar WB, et al. High dietary sodium intake impairs endothelium-dependent dilation in healthy salt-resistant humans. J Hypertens. 2013 Mar; 31(3):530-6.

7 Roustit M, Cracowski JL. Non-invasive assessment of skin microvascular function in humans: an insight into methods. Microcirculation. 2012 Jan;19(1):47-64. 


\begin{tabular}{l|l}
\hline Kidney Blood Press Res 2019;44:835-847 \\
\hline DOI: 10.1159/000501747 & $\begin{array}{l}\text { (c) 2019 The Author(s). Published by S. Karger AG, Basel } \\
\text { www.karger.com/kbr }\end{array}$ \\
\hline
\end{tabular}

Barić et al.: HS Diet and Microvascular Function

8 Cavka A, Cosic A, Jukic I, Jelakovic B, Lombard JH, Phillips SA, et al. The role of cyclo-oxygenase-1 in high-salt diet-induced microvascular dysfunction in humans. J Physiol. 2015 Dec;593(24):5313-24.

9 Heer M, Frings-Meuthen P, Titze J, Boschmann M, Frisch S, Baecker N, et al. Increasing sodium intake from a previous low or high intake affects water, electrolyte and acid-base balance differently. Br J Nutr. 2009 May; 101(9):1286-94.

10 Larsen SC, Ängquist L, Sørensen TI, Heitmann BL. 24h urinary sodium excretion and subsequent change in weight, waist circumference and body composition. PLoS One. 2013 Jul;8(7):e69689.

11 Sagnella GA, Markandu ND, Buckley MG, Miller MA, Singer DR, MacGregor GA. Hormonal responses to gradual changes in dietary sodium intake in humans. Am J Physiol. 1989 Jun;256(6 Pt 2):R1171-5.

12 Singer DR, Markandu ND, Buckley MG, Miller MA, Sagnella GA, Lachno DR, et al. Blood pressure and endocrine responses to changes in dietary sodium intake in cardiac transplant recipients. Implications for the control of sodium balance. Circulation. 1994 Mar;89(3):1153-9.

13 Jelakovic B, Premuzic V, Cvoriscec D, Erceg I, Fucek M, Jelakovic M, et al. Salt Mapping in Croatia. Croatian Action on Salt and Health (CRASH). Kidney Blood Press Res. 2009;32:323.

14 Kotchen TA, Cowley AW Jr, Frohlich ED. Salt in health and disease-a delicate balance. N Engl J Med. 2013 Mar; 368(13):1229-37.

15 Stupin M, Stupin A, Rasic L, Cosic A, Kolar L, Seric V, et al. Acute exhaustive rowing exercise reduces skin microvascular dilator function in young adult rowing athletes. Eur J Appl Physiol. 2018 Feb;118(2):461-74.

16 Lenasi H, Strucl M. The effect of nitric oxide synthase and cyclooxygenase inhibition on cutaneous microvascular reactivity. Eur J Appl Physiol. 2008 Aug;103(6):719-26.

17 Widmer RJ, Lerman A. Endothelial dysfunction and cardiovascular disease. Glob Cardiol Sci Pract. 2014 Oct; 2014(3):291-308.

18 Townsend N, Wilson L, Bhatnagar P, Wickramasinghe K, Rayner M, Nichols M. Cardiovascular disease in Europe: epidemiological update 2016. Eur Heart J. 2016 Nov;37(42):3232-45.

19 Elliott P, Stamler J, Nichols R, Dyer AR, Stamler R, Kesteloot H, et al.; Intersalt Cooperative Research Group. Intersalt revisited: further analyses of 24 hour sodium excretion and blood pressure within and across populations. BMJ. 1996 May;312(7041):1249-53.

20 The Trials of Hypertension Prevention Collaborative Research Group. Effects of weight loss and sodium reduction intervention on blood pressure and hypertension incidence in overweight people with high-normal blood pressure. The Trials of Hypertension Prevention, phase II. Arch Intern Med. 1997 Mar;157(6):657-67.

21 Sacks FM, Svetkey LP, Vollmer WM, Appel LJ, Bray GA, Harsha D, et al.; DASH-Sodium Collaborative Research Group. Effects on blood pressure of reduced dietary sodium and the Dietary Approaches to Stop Hypertension (DASH) diet. N Engl J Med. 2001 Jan;344(1):3-10.

22 Drenjancevic-Peric I, Frisbee JC, Lombard JH. Skeletal muscle arteriolar reactivity in SS.BN13 consomic rats and Dahl salt-sensitive rats. Hypertension. 2003 May;41(5):1012-5.

23 Durand MJ, Moreno C, Greene AS, Lombard JH. Impaired relaxation of cerebral arteries in the absence of elevated salt intake in normotensive congenic rats carrying the Dahl salt-sensitive renin gene. Am J Physiol Heart Circ Physiol. 2010 Dec;299(6):H1865-74.

24 Boegehold MA, Drenjancevic I, Lombard JH. Salt, Angiotensin II, Superoxide, and Endothelial Function. Compr Physiol. 2015 Dec;6(1):215-54.

25 Dishy V, Sofowora GG, Imamura H, Nishimi Y, Xie HG, Wood AJ, et al. Nitric oxide production decreases after salt loading but is not related to blood pressure changes or nitric oxide-mediated vascular responses. J Hypertens. 2003 Jan;21(1):153-7.

26 Tzemos N, Lim PO, Wong S, Struthers AD, MacDonald TM. Adverse cardiovascular effects of acute salt loading in young normotensive individuals. Hypertension. 2008 Jun;51(6):1525-30.

27 Greaney JL, DuPont JJ, Lennon-Edwards SL, Sanders PW, Edwards DG, Farquhar WB. Dietary sodium loading impairs microvascular function independent of blood pressure in humans: role of oxidative stress. J Physiol. 2012 Nov;590(21):5519-28.

28 Priestley JR, Buelow MW, McEwen ST, Weinberg BD, Delaney M, Balus SF, et al. Reduced angiotensin II levels cause generalized vascular dysfunction via oxidant stress in hamster cheek pouch arterioles. Microvasc Res. 2013 Sep;89:134-45.

29 Durand MJ, Lombard JH. Low-dose angiotensin II infusion restores vascular function in cerebral arteries of high salt-fed rats by increasing copper/zinc superoxide dimutase expression. Am J Hypertens. 2013 Jun;26(6): 739-47.

30 Cosic A, Jukic I, Stupin A, Mihalj M, Mihaljevic Z, Novak S, et al. Attenuated flow-induced dilatation of middle cerebral arteries is related to increased vascular oxidative stress in rats on a short-term high salt diet. J Physiol. 2016 Sep;594(17):4917-31.

31 Weber DS, Lombard JH. Elevated salt intake impairs dilation of rat skeletal muscle resistance arteries via ANG II suppression. Am J Physiol Heart Circ Physiol. 2000 Feb;278(2):H500-6.

32 Zhu J, Drenjancevic-Peric I, McEwen S, Friesema J, Schulta D, Yu M, et al. Role of superoxide and angiotensin II suppression in salt-induced changes in endothelial Ca2+ signaling and NO production in rat aorta. Am J Physiol Heart Circ Physiol. 2006 Aug;291(2):H929-38.

33 Cavka A, Cosic A, Grizelj I, Koller A, Jelaković B, Lombard JH, et al. Effects of AT1 receptor blockade on plasma thromboxane A2 (TXA2) level and skin microcirculation in young healthy women on low salt diet. Kidney Blood Press Res. 2013;37(4-5):432-42. 
34 Mak GS, Sawaya H, Khan AM, Arora P, Martinez A, Ryan A, et al. Effects of subacute dietary salt intake and acute volume expansion on diastolic function in young normotensive individuals. Eur Heart J Cardiovasc Imaging. 2013 Nov;14(11):1092-8.

35 Titze J. Sodium balance is not just a renal affair. Curr Opin Nephrol Hypertens. 2014 Mar;23(2):101-5.

36 Schneider MP, Raff U, Kopp C, Scheppach JB, Toncar S, Wanner C, et al. Skin Sodium Concentration Correlates with Left Ventricular Hypertrophy in CKD. J Am Soc Nephrol. 2017 Jun;28(6):1867-76.

37 Hofmeister LH, Perisic S, Titze J. Tissue sodium storage: evidence for kidney-like extrarenal countercurrent systems? Pflugers Arch. 2015 Mar;467(3):551-8.

38 Selvarajah V, Mäki-Petäjä KM, Pedro L, Bruggraber SF, Burling K, Goodhart AK, et al. Novel Mechanism for Buffering Dietary Salt in Humans: Effects of Salt Loading on Skin Sodium, Vascular Endothelial Growth Factor C, and Blood Pressure. Hypertension. 2017 Nov; 70(5):930-7.

39 Oberleithner H. Sodium selective erythrocyte glycocalyx and salt sensitivity in man. Pflugers Arch. 2015 Jun; 467(6):1319-25.

40 Oberleithner H, Wilhelmi M. Salt Sensitivity Determined From Capillary Blood. Kidney Blood Press Res. 2016; 41(4):355-64.

41 Oberleithner H, Peters W, Kusche-Vihrog K, Korte S, Schillers H, Kliche K, et al. Salt overload damages the glycocalyx sodium barrier of vascular endothelium. Pflugers Arch. 2011 Oct;462(4):519-28. 